\title{
Implications of Electrical Impedance-Based
}

\section{Microbiological Technology in Pork Meat Processing} Industry for the Rapid Detection and Quantification of Salmonella Spp.

\author{
Cristina Pablos, Javier Marugán, Sandra Cristóbal and Rafael van Grieken \\ Department of Chemical and Environmental Technology, University Rey Juan Carlos, C/Tulipán s/n, Móstoles (Madrid) 28933, \\ Spain
}

\begin{abstract}
The absence of efficient tools for preventing bacterial contamination in the meat processing industry as well as for detecting Salmonella positive samples in real time is a matter of concern. Impedance technology has proved its effectiveness as a bacterial quantification tool for research purposes instead of laborious standard plate count, and as a detection tool to substitute tedious current horizontal method ISO 6579:2002. Calibration curves were carried out for S. enteritidis and S. typhimurium in raw pork matrix $\left(\mathrm{R}^{2}>0.90\right)$. Calibrations of mixtures of both strains at different ratio were prepared, showing a high efficiency to differentiate bacterial metabolism. Impediometry was also validated against standard plate count in raw pork samples treated by UV-C illumination to inactivate Salmonella. Even, damaged but still viable bacteria were recorded. Detection of Salmonella by impediometry led to a decrease in false positives, obtaining results within $30 \mathrm{~h}$ compared to $72 \mathrm{~h}$ in case of conventional method.
\end{abstract}

Key words: Impediometry, S. enteritidis, S. typhimurium, quantification, detection, pork meat.

\section{Introduction}

The high number of outbreaks of food- and water-borne diseases caused by pathogenic bacteria indicates the importance of food and water safety. Salmonella is considered to be one of the most common and virulent foodborne pathogen. The ingestion of this microorganism in contaminated food or water may lead to salmonellosis, a zoonotic disease associated with gastroenteritis and fevers. It is responsible for ca. 1.4 million human Salmonella infections in the United States [1,2] and 176,395 reported human cases in the European Union [3]. Two different strains of Salmonella have been identified as the main responsible for human illnesses, S. enteritidis and S. typhimurium.

Corresponding author: Javier Marugán, professor, research fields: advanced oxidation technologies, modelling of kinetics of bacterial inactivation.
Over the past decade, regulations $[4,5]$ have laid down acceptable microbiological criteria for meat-based products, requiring the absence of Salmonella per $25 \mathrm{~g}$ of meat. Thus, food safety is becoming a major concern for pork meat since positive samples have been detected up to $18 \%$ [3]. The most critical points of microbial contamination correspond to the slaughter and carcass handling together with cross-contamination on surfaces such as conveyor belts in food processing industries [3]. In addition, ready-to-eat (RTE) or processed meat products such as ground meat are more and more demanded by consumers. This trend together with the fact that these products are not fully cooked before their consumption increases the risk of food-borne diseases.

Traditional culture method for Salmonella spp. detection in food, according to the current ISO 6579:2002 Standard [6], consists of non-selective 

Industry for the Rapid Detection and Quantification of Salmonella Spp.

pre-enrichment, selective enrichment, plating on selective agars, and eventually, biochemical and serological confirmation. Despite ISO 6579:2002 Standard is widely used and serves as reference to compare with other techniques, it is labour-intensive and time-consuming, requiring 3-4 days for presumptive results and 5-7 days for confirmation [1, 7]. Therefore, it is impossible to obtain a real time response. When the results are obtained, the product has already been released for sale. This fact demonstrates the lack of proper controls of the presence of Salmonella in food. As a result, several strategies are being developed for a better control of foodborne pathogens.

As a strategy, over the past decades, numerous rapid methods have been developed to shorten the assay time and increase its sensitivity [1,8]. Moreover, since pathogenic distribution is irregular in the final product and a high throughput screening is required for a large number of samples, there may be a tool for fast screening to identify positive samples, allowing the release of negative samples to the market.

To date, the most widely used commercial tests correspond to either immunoassays such as enzyme linked immunosorbent assays (ELISA) or DNA based methods such as polymerase chain reaction (PCR). Although they have notably reduced the assay time and improved the detection limit, they also show some shortcomings including long times of pre- or postenrichment [7, 9]; cross or inhibited reactions by compounds or background flora from some food matrices; variation of antigens and DNA of the target pathogen [1, 7-10]. Moreover, ELISA shows low suitability in the binding between Salmonella and its antibodies, whereas PCR involves far away more costs, labour-intensive tasks [11] and cannot distinguish between death, supposedly non-pathogenic, and viable cells.

Impedance technology is a rapid method which measures changes in electrical impedance in a medium induced by bacterial metabolism due to the release of ionic metabolites from live cells. These metabolites are responsible for the decrease in the impedance of the medium [2, 12-14]. After the important work of Torry Research Station [15] in 1978 and Eden and Eden [16] in 1984, and Owens et al. [17] in 1989 reporting the principle of indirect impedance, several efforts have been made on the detection of Salmonella by impedance-based technology in food samples [9, 12, 13, 18-23].

Concerning food hygiene, new emerging non-thermal technologies such as UV-C illumination have started to be developed in recent years [24, 25]. These emerging technologies may prompt to reduce microbial cross contamination in food processing industries, extending the shelf-life of the fresh product, and allowing it to keep its natural appearance, flavour and taste. However, these kinds of studies require intensive experimentation and generation of a large number of data. Impedance would be suitable for carrying out this kind of application, since it distinguishes between dead and viable cells and reduces working time, materials and samples compared to classic microbiology. Moreover, impedance technology may not only reduce the time assay but may also be a potentially powerful and reliable tool to understand microbial growth and other prevention factors [26-28].

Thus, the aim of this work is to validate electrical impedance-based technology for a rapid detection of Salmonella in raw pork meat against the conventional current ISO horizontal method. Moreover, the use of electrical impedance-based technology as an alternative to the standard plate count technique for the quantification of Salmonella is also addressed especially for food safety research purposes.

\section{Material and Methods}

\subsection{Salmonella Culture, Media and Growth Conditions}

Salmonella strains were provided lyophilized by the Spanish Type Culture Collection. Fresh cultures of Salmonella enteritidis (CECT 4300, corresponding to 

Industry for the Rapid Detection and Quantification of Salmonella Spp.

ATCC 13076) and Salmonella typhimurium (CECT 4594, ATCC 14028) were grown in buffered peptone water (BPW, Scharlab) at $37{ }^{\circ} \mathrm{C}$ under rotary shaking for $24 \mathrm{~h}$. Fresh bacterial cultures of around $10^{9}$ Colony Forming Units $(\mathrm{CFU}) / \mathrm{mL}$ of stationary concentration were obtained. The cells were pelleted by centrifuging at 3,500 rpm for $25 \mathrm{~min}$ and resuspended in sterilized buffered peptone water. Serial dilutions were prepared in sterilized buffered peptone water as well for further applications. Escherichia coli K-12 (CECT 4624, ATCC 23631) and Enterococcus faecalis (CECT 5143, ATCC 11700) were grown in the same way for further purposes.

\subsection{Electrical Impedance Measurements}

A $\mu$-Trac 4200 (SY-LAB) system was used for recording impedance changes in the growth medium throughout time every 5 min. Modified selenite-cystine medium (Bi205A, SY-LAB) $(9.9 \mathrm{~mL})$ was used in this system as selective culture medium for Salmonella. In all the cases, the measuring plastic tubes containing the medium were inoculated with 0.1 $\mathrm{mL}$ of diluted suspensions of Salmonella from an overnight culture. The tubes were tightly closed and placed in the $\mu$-Trac 4200 apparatus and monitored for $24 \mathrm{~h}$ as maximum time. The temperature in the incubator was controlled at $37{ }^{\circ} \mathrm{C}$. The impedance relative changes in the medium ( $\left.\begin{array}{ll}\% & \mathrm{M}\end{array}\right)$ were automatically recorded during incubation in the measuring system. The threshold value, $10 \%$, represented the decrease of the initial value of electrical impedance due to the bacterial metabolic activity. The detection time (DT) needed for the M-value of each sample to exceed the threshold value was evaluated since it corresponds to sharp and significant changes in the impedance of the bacterial growth medium.

\subsection{Salmonella Quantification Assay}

2.3.1 Calibration Curve Preparation

Separate calibration curves were prepared for the quantification of S. enteritidis and S. typhimurium and analysed by $\mu$-Trac and the standard plate count technique using deoxycholate citrate agar (DCA, Fluka) as selective agar. Raw pieces of pork meat weighting about $25 \mathrm{~g}$ in $225 \mathrm{~mL}$ buffered peptone water were used as matrix. This fresh raw pork meat was provided by Embutidos La Nuncia S.L., a local supplier in Spain, and kept at $4{ }^{\circ} \mathrm{C}$ for a maximum of 4 days. Thus, 15 different batches of raw pork meat were tested in total. To assess the accuracy and reproducibility of impedance data, the relationship between multiple inoculation levels and DT was examined. Moreover, two different ways of preparing the calibration curves were carried out:

(1) Calibration 1: The calibration was performed by preparing serial dilutions (from $10^{8}$ up to $10^{-1}$ $\mathrm{CFU} / \mathrm{mL}$ ) of each microorganism, S. enteritidis and $S$. typhimurium, from the bacterial suspension mentioned in 2.1. whose concentration corresponded to $10^{9}$ $\mathrm{CFU} / \mathrm{mL}$. Thus, $0.1 \mathrm{~mL}$ of each dilution was inoculated into a plastic tube and measured in the $\mu$-Trac 4200 apparatus.

Additional calibration curves were carried out by mixing both strains. After centrifugation and resuspension up to $5 \mathrm{~mL}$ with sterilized BPW for each strain separately, 3 vials containing both strains mixed in a different ratio were prepared. The different ratios tested corresponded to 20:80, 50:50; and 80:20\% v/v of $S$. typhimurium and $S$. enteritidis, respectively. Serial dilutions were prepared from each vial and electrical impedance changes of the growth medium were measured as explained above.

(2) Calibration 2: Different concentration of $S$. enteritidis was inoculated onto the surface of $25 \mathrm{~g}$ of raw pork meat. The cut pieces of meat were inoculated with a load of Salmonella of $10^{8}$ up to $10^{2} \mathrm{CFU}$ and spotted with $100 \mu \mathrm{L}$ taken from the fresh culture previously centrifuged and the serial dilutions prepared from it as mentioned above. They were allowed to dry for about $20 \mathrm{~min}$ in a microbiological safety cabinet. Then, the samples were homogenized 

Industry for the Rapid Detection and Quantification of Salmonella Spp.

in $225 \mathrm{~mL}$ of sterile buffered water peptone in a sterile stomacher bag with a stomacher Bag Mixer 400 (Scharlab) for $2 \mathrm{~min}$. One tenth of a milliliter from each sample was added into a plastic tube and measured in the $\mu$-Trac 4200 apparatus.

In both cases, non-inoculated growth medium and matrix, and BPW used as diluent, were used as controls of background values. At the same time, the viable bacterial concentration of Salmonella was determined by conventional plating method performed by plating $0.1 \mathrm{~mL}$ of appropriate dilutions onto DCA selective agar. Colonies were counted after incubation at $37^{\circ} \mathrm{C}$ for $24 \mathrm{~h}$.

The concentration of viable Salmonella obtained by standard plate count technique for the calibration 1 was reported in $\mathrm{CFU} / \mathrm{mL}$. To be able to include these experimental points in a final calibration in $\mathrm{CFU} / \mathrm{g}$, the former values were multiplied by $9 \mathrm{~mL} / \mathrm{g}$ (considering the dilution 1:9 sample/BPW made in the calibration 2). Eventually, a regression line of logarithmic bacterial concentration against detection time (DT) was calculated to determine the correlation between DT and concentration of viable Salmonella (CFU/g).

$$
\log _{10} \mathrm{C}(\mathrm{CFU} / \mathrm{g})=\mathrm{a}+\mathrm{b} \cdot \mathrm{DT}(\mathrm{h})
$$

Each calibration was independently repeated for 6 times. In every experiment, each concentration point was repeated in duplicate.

\subsubsection{Determination of Sensitivity}

Once the $\mu$-Trac 4200 apparatus was calibrated for this type of food sample, data of viable concentration of $S$. enteritidis obtained by impedance measurements were evaluated in comparison with conventional microbiology. Raw pork samples, inoculated with Salmonella and illuminated by UV-C as emerging technology to reduce bacterial contamination were used for this purpose.

The UV-C illumination device consisted of three Osram HNS 6W lamps, with an emission peak at 254 $\mathrm{nm}$, and a light flux, determined by a Delta Ohm HD 2102.2 radiometer of $42 \mathrm{~W} / \mathrm{m}^{2}$. The UV-C lamps were enclosed in a wooden box $(23.5 \times 13.5 \times 18 \mathrm{~cm})$ covered with aluminum foil and placed $18 \mathrm{~cm}$ above the samples. No noticeable increase in temperature was measured inside the set-up due to the treatment.

Raw pork pieces of meat weighting about $25 \mathrm{~g}$ were cut, placed onto sterilised glass Petri plates, inoculated with loads of $S$. enteritidis of $10^{6}$ and $10^{4} \mathrm{CFU}$, and left to dry. After inoculation, meat pieces were exposed to UV-C light treatments for 5, 15 and $30 \mathrm{~min}$. Non-radiated inoculated-pork meat pieces were considered as control of initial concentration of Salmonella before the treatment. Non-radiated non-inoculated-pork meat pieces were considered as a control of the bacterial background value. All experiments were conducted in duplicate and quantified in the same day of the UV-C treatment. Samples were homogenized in $225 \mathrm{~mL}$ of sterile BPW with a stomacher Bag Mixer 400 for $2 \mathrm{~min}$. Concentration of viable Salmonella present in meat was quantified by both, the conventional method, based on dilution, plating on DCA agar and incubation at $37{ }^{\circ} \mathrm{C}$ for $24 \mathrm{~h}$; and the impedance technology, based on adding $0.1 \mathrm{~mL}$ of the sample into plastic tubes to be measured by $\mu$-Trac 4200 . Experiments were repeated in triplicate.

The sensitivity of the impedance-based measurements compared to classic microbiology for quantifying viable concentration of $S$. enteritidis in raw pork meat $(\mathrm{CFU} / \mathrm{g})$ was analysed. The potential effect of UV-C illumination to reduce Salmonella in meat was evaluated by quantifying the concentration of viable Salmonella before and after the illumination.

\subsection{Salmonella Detection Assay}

The validation of this impedance-based technology for the detection of Salmonella was carried out against the traditional method (ISO 6579:2002 Standard) for 15 samples of raw pork meat. Despite 20 samples are required to validate a detection method according to ISO 16140:2003 Standard [29], this value of samples would be acceptable for studying the potential of 

Industry for the Rapid Detection and Quantification of Salmonella Spp.

impedance-based technology in this application. Food samples of ca. $25 \mathrm{~g}$ were homogenised in $225 \mathrm{~mL}$ of buffered peptone water and pre-enriched under rotary shaking for $6-8 \mathrm{~h}$ at $37^{\circ} \mathrm{C}$. Following this, $0.1 \mathrm{~mL}$ of the pre-enrichment culture was transferred to $9.9 \mathrm{~mL}$ of the growth medium to be measured by impediometry at $37^{\circ} \mathrm{C}$ for $24 \mathrm{~h}$ as maximum time. To compare the result to the conventional method, the pre-enrichment step was conducted up to $24 \mathrm{~h}$, followed by a selective enrichment of $0.1 \mathrm{~mL}$ in Rappaport Vassiliadis (RVS, Scharlab) and $1 \mathrm{~mL}$ in Muller-Kauffmann Tetrathionate-Novobiocin (MKTTn, Panreac), being the final volume of $10 \mathrm{~mL}$, for $24 \mathrm{~h}$ at $42{ }^{\circ} \mathrm{C}$ and $37{ }^{\circ} \mathrm{C}$ respectively. The selective enrichment media were streaked on solid selective media, xylose-lysine-deoxycholate agar (XLD, Scharlab) and DCA, for $24 \mathrm{~h}$ at $37{ }^{\circ} \mathrm{C}$ to isolate presumptive positive Salmonella colonies. Presumptive Salmonella colonies isolated on plating media were incubated in Tryptic Soy Agar (TSA, Scharlab) for $24 \mathrm{~h}$ at $37{ }^{\circ} \mathrm{C}$ followed by biochemical confirmation with Enteropluri-Test $\AA$. This test permitted the confirmation of these presumptive Salmonella colonies. Positive results obtained by impediometry were also confirmed by this test. Enteropluri-Test ${ }^{\circledR}$ is based on the inoculation of the microorganism to be identified in 15 special culture media, allowing the execution of 15 biochemical reactions. Microorganisms were identified evaluating the colour change of the different culture media after 18-24 h of incubation at $37{ }^{\circ} \mathrm{C}$. The combination of positive and negative reactions allowed building up a code number to identify bacteria by using a Codebook supplied by Enteropluri-Test ${ }^{\circledR}$.

The sensitivity of impediometry for the detection of Salmonella in raw pork meat was checked by carrying out the conventional and alternative method by inoculating food samples, previously to their pre-enrichment, with a load of $S$. enteritidis and $S$. typhimurium corresponding to $10^{4} \mathrm{CFU}$. Its specificity was also confirmed by inoculating a load of E. coli and E. faecalis of $10^{6} \mathrm{CFU}$ onto two food samples respectively.

\section{Results and Discussion}

\subsection{Salmonella Quantification Performance in Raw Pork Meat Samples by Impedance Measurements}

3.1.1 Correlation between Detection Time and Concentration of Salmonella

Fig. 1 shows typical impedance growth curves for different initial concentration of $S$. enteritidis ranging from $10^{8}$ to $10^{1} \mathrm{CFU} / \mathrm{mL}$. Microbial metabolism produces lower molecular, charged decomposition products, leading to a decrease in the electrical impedance of the growth medium. These relative electrical impedance changes $(\% \mathrm{M})$ are recorded throughout the incubation time.

Regarding the shape of the impedance growth curves, three regions are observed according to other authors [2, 20, 22, 27, 30]: (1) an initial region where the impedance change is hardly affected. Bacteria are adapting their metabolism to the nutrients present in the growth medium, so bacterial population is not high enough or their growth rates are not fast enough to produce detectable changes in impedance; (2) the second region where the impedance change sharply increases, since microorganisms have reached a concentration of ca. $10^{6}-10^{7} \mathrm{CFU} / \mathrm{mL}$, starting to proliferate, rising linearly; (3) and the third region, where the impedance change remains practically parallel to the time axis again, since microorganisms cease their metabolism as all the resources in the medium have been metabolized to an end product, and there is also a depletion of nutrients. It clearly matches a typical shape of a bacterial growth curve, which includes the lag phase where bacteria are metabolizing but not multiplying, the exponential growth phase where bacteria multiply exponentially, and the stationary phase where the bacterial cell number remains relatively constant.

To consider a positive detection, a threshold value of $10 \%$, as shown in Fig. 1, has to be exceeded by the 


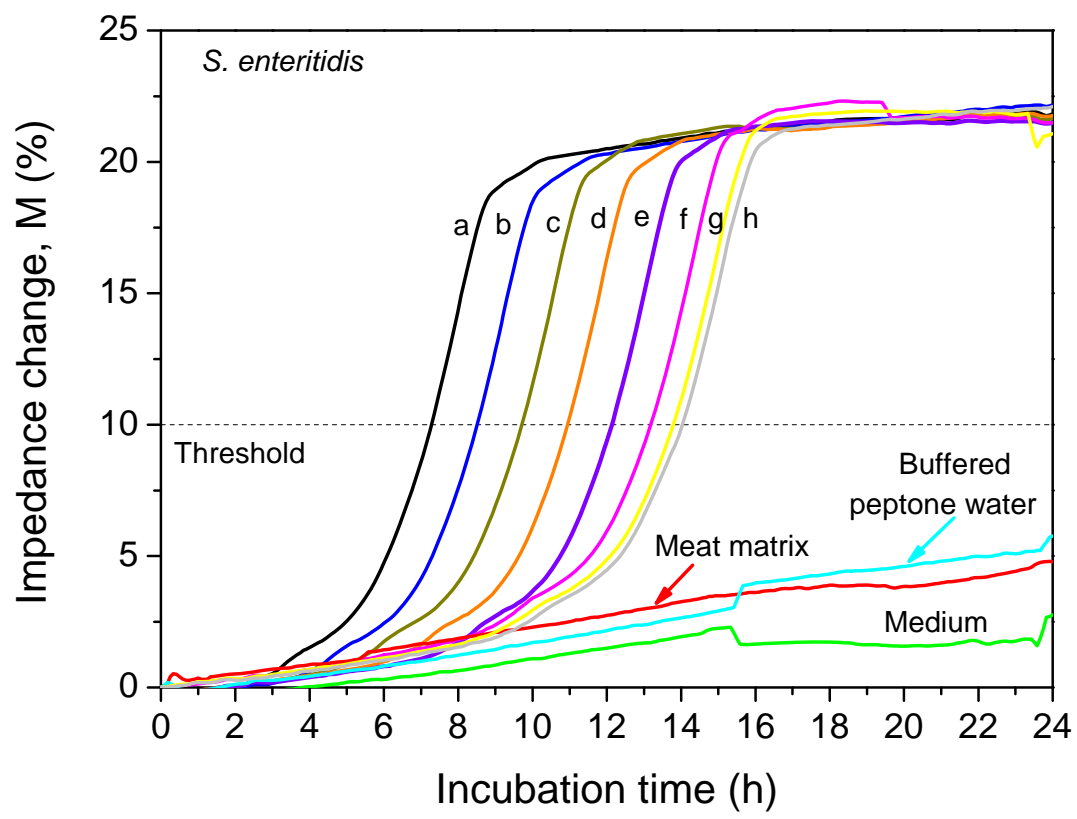

Fig. 1 Typical impedance growth curves obtained from samples containing different initial concentration of $S$. enteritidis according to the 1st method of calibration detailed in 2.3.1. Curve (a) $1.1 \times 10^{8}$; (b) $1.2 \times 10^{7}$; (c) $1.3 \times 10^{6}$, (d) $1.4 \times 10^{5}$; (e) 1.1 $\times 10^{4}$; (f) $9.6 \times 10^{2}$, (g) $1.2 \times 10^{2}$; (h) $6.1 \times 10^{1} \mathrm{CFU} / \mathrm{mL}$ diluted from a stock solution $\left(1 \times 10^{9} \mathrm{CFU} / \mathrm{mL}\right)$.

impedance curve. The incubation time required to reach the threshold value is called detection time (DT). This point of inflection of the curve and also the earlier exponential phase has the most relevance for this measuring technology. It indicates a sharp decrease in the initial value of the impedance in the growth medium.

The control samples, which contain no bacteria, such as the medium, raw pork meat matrix and BPW, show no change in impedance change, indicating that there is no ionic release resulted from bacterial metabolism in the medium throughout the incubation time. All the samples containing $S$. enteritidis reach a point where the rate of change in impedance starts to change, corresponding to the DT. It is also seen that the detection time is related to the initial bacterial concentration in the medium. Thus, the higher the initial concentration of Salmonella is, the shorter the detection time, and the earlier the results will be obtained [2, 26, 27, 30]. Therefore, according to Fig. 1, results are available within only a few hours $(6 \mathrm{~h})$ for the most contaminated sample $\left(1.1 \times 10^{8} \mathrm{CFU} / \mathrm{mL}\right)$. Moreover, the delay in detection time for serial dilutions of the initial sample allows predicting an approximated value of bacterial concentration present in the sample.

Unlike the standard plate count technique, impedance technology allows the detection of live cells in a dynamic way. It not only avoids time-consuming plate counts, but also monitors the bacterial growth and metabolism in real time, which allows determining the metabolic capacities of proliferating microorganisms, their level of activity and their pathogenic potential on food from the beginning of the analysis.

Fig. 2 shows the calibration curve obtained for $S$. enteritidis in raw pork meat by plotting the logarithmic values of initial concentration of Salmonella against the detection time. It must be noticed that the overlapped points from both calibrations follow the same linear trend. It agrees with similar values of slope and intercept obtained from each method of calibration considering a linear regression model.

The detection time is inversely proportional to the initial cell number as observed in the impedance growth 

Industry for the Rapid Detection and Quantification of Salmonella Spp.

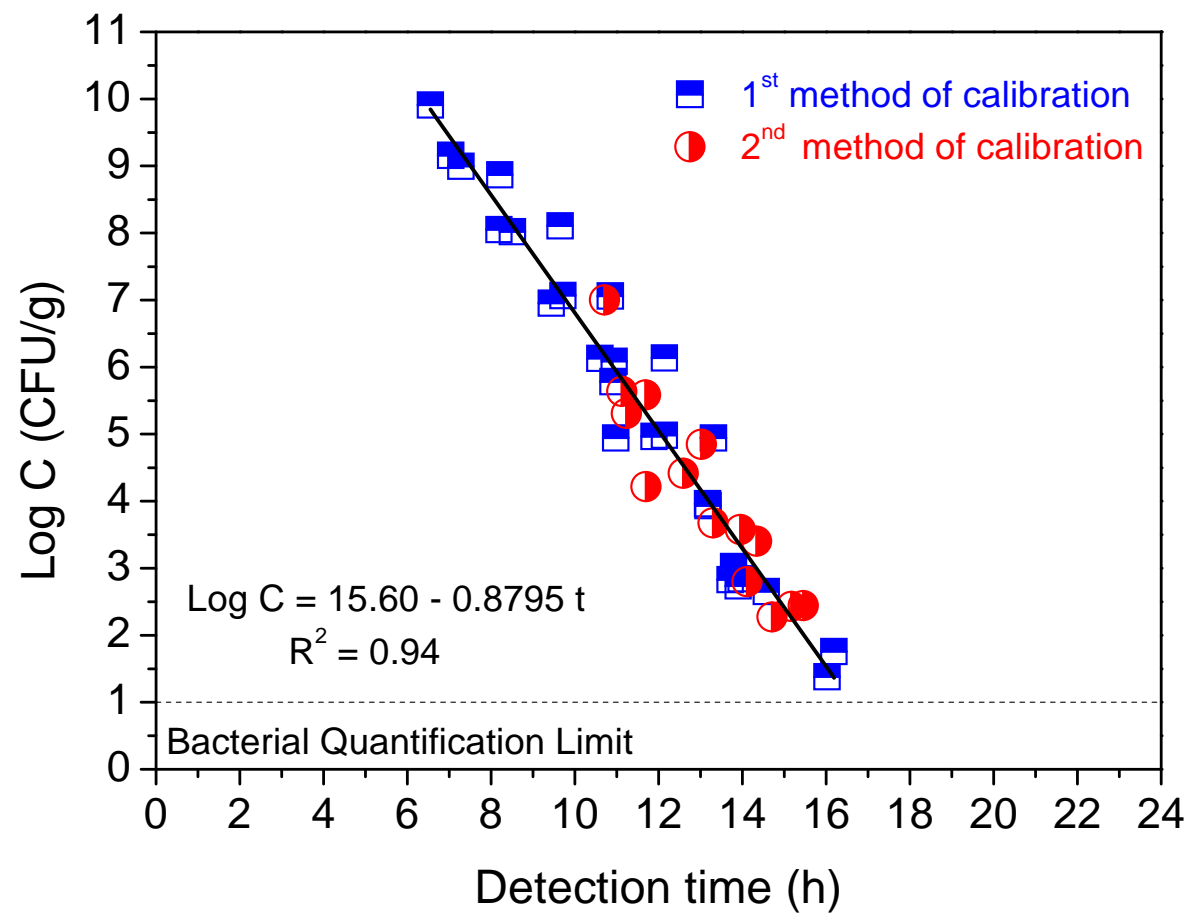

Fig. 2 Effect of calibration method: Logarithmic values of initial concentration of $S$. enteritidis ranging from $10^{10}$ to $10^{1}$ CFU/g as a function of detection time obtained from the impedance growth curves. Regression equations obtained for each calibration method corresponded to: $\log C=15.70-0.8884 t\left(R^{2}=0.95\right)\left(1\right.$ st method) and $\log C=15.07-0.8380 t\left(R^{2}=0.86\right)$ (2nd method).

curves. A linear relation between the detection times and the initial cell number in terms of logarithmic values, determined by plate count, are obtained, as was pinpointed by Fehlhaber and Krüger [26], Ruan et al. [11], Yang et al. [30], Yang and Bashir [2], and Johnson et al. [27]. A value of $\mathrm{R}^{2}>0.9$ is achieved after fitting a regression line by the method of least-squares considering all the experimental data, indicating a strong linear correlation between initial Salmonella concentration and DT. Moreover, the impedance method does not become more erratic for meat samples containing lower concentration of Salmonella $\left(<2 \log _{10} \mathrm{CFU} / \mathrm{g}\right)$ unlike what was pointed out by Russell [14]. Thus, the detection time of the impedance method for the quantification of $S$. enteritidis in raw pork meat ranges from about $6.05 \mathrm{~h}$ to $16.05 \mathrm{~h}$ for initial bacterial concentration of $10^{10}$ $\mathrm{CFU} / \mathrm{g}$ to $10^{1} \mathrm{CFU} / \mathrm{g}$ respectively.

The quantification and detection limits of Salmonella correspond to $10^{1} \mathrm{CFU} / \mathrm{g}$ and $1 \mathrm{CFU} / \mathrm{g}$ respectively. Therefore, a low detection limit is achieved by impedance technology in Salmonella quantification assays. This fact may reduce pre-enrichment period of meat in Salmonella detection assays compared to the traditional method, together with an improvement in efficiency of the method since the demand for detection limit in raw pork meat is less than 1 cell per $25 \mathrm{~g}$ of food.

3.1.2 Effect of the Kind of Bacterial Strain on the Detection Time. Comparison of Bacterial Growth Rates

Fig. 3 shows that linear responses have also been found between detection times obtained from impedance bacterial growth curves and initial concentration of S. typhimurium in the range of $10^{1}$ $\mathrm{CFU} / \mathrm{g}$ to $10^{10} \mathrm{CFU} / \mathrm{g}$. Thus, this calibration can be used for quantification of bacterial cells.

It is worth noting that the detection time is approximately $11 \mathrm{~h}$ and $21.5 \mathrm{~h}$ for $10^{10} \mathrm{CFU} / \mathrm{g}$ and $10^{1}$ $\mathrm{CFU} / \mathrm{g}$ of viable $S$. typhimurium respectively. So, a longer 


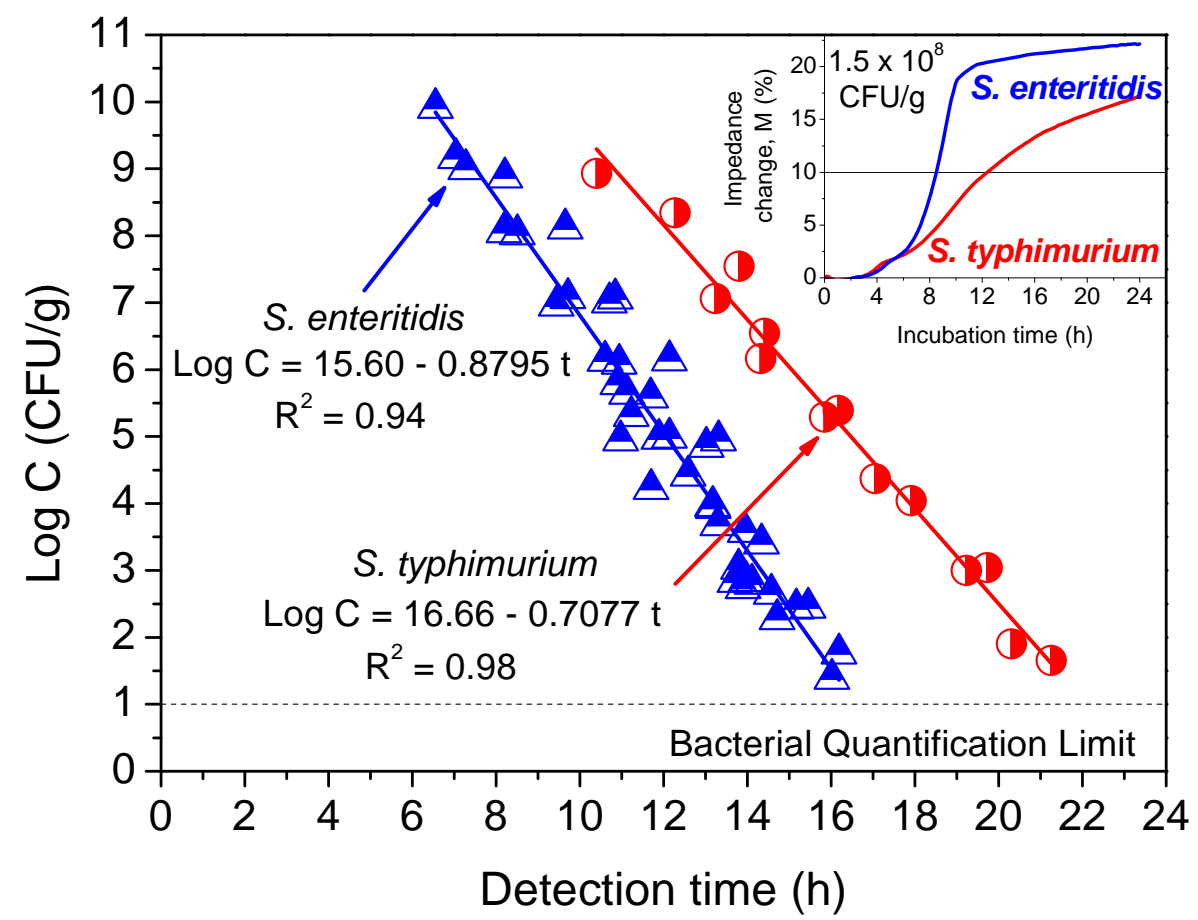

Fig. 3 Comparison of linear response between detection times and logarithmic values of initial concentration of two different strains of Salmonella: S. enteritidis and S. typhimurium. Upper inset: Impedance bacterial growth curves for both strains of Salmonella which correspond to the same value of initial concentration $\left(1.5 \times 10^{8} \mathrm{CFU} / \mathrm{g}\right)$.

incubation time is required for quantification of viable S. typhimurium compared to that of $S$. enteritidis. It agrees with the flat nature of the impedance growth curve of S. typhimurium shown by Fig. 3, upper inset, indicating a delay for a significant change in impedance, the DT, to takes place. Therefore, DT not only depends on the initial concentration of bacteria but also on the bacterial growth rate, being specific for each strain. This fact agrees with Johnson et al. [27], who stated that the slope of the DT line taken over multiple inoculation levels gives a direct measurement of the bacterial growth rate. It is also confirmed by the slope value obtained from the calibration curves for each strain. S. enteritidis reveals a higher value of slope in comparison with $S$. typhimurium, which would indicate a faster bacterial growth rate for the former. This fact obliges to carry out a calibration for each strain with quantification purposes. As a result, since bacteria may be stressed by compounds present in either growth medium or matrix, a new calibration would be required for each matrix and growth medium to be tested [22].

3.1.3 Effect of the Mixture of Bacterial Strainson the Detection Time. Comparison of Bacterial Growth Rates

Fig. 4 depicts how the presence of two strains of Salmonella, with different growth rate, mixed in different ratio influences the overall bacterial growth rate and DT. Three different levels of initial concentration are shown in Fig. 4.

As the initial concentration of Salmonella increases, a lower difference among impedance growth curves is observed. In addition, the DT given by the impedance growth curves becomes more similar to that of pure $S$. enteritidis when both strains are mixed, independently of the ratio. It seems that impediometry is detecting impedance changes in growth medium for the bacteria with a faster metabolism and growth rate. In fact, when all the inoculation levels tested are plotted against detection time (Fig. 5), a decrease in DT is seen for all the mixtures of bacteria. As a result, overall time of analysis is reduced. It is also confirmed 
Implications of Electrical Impedance-Based Microbiological Technology in Pork Meat Processing Industry for the Rapid Detection and Quantification of Salmonella Spp.
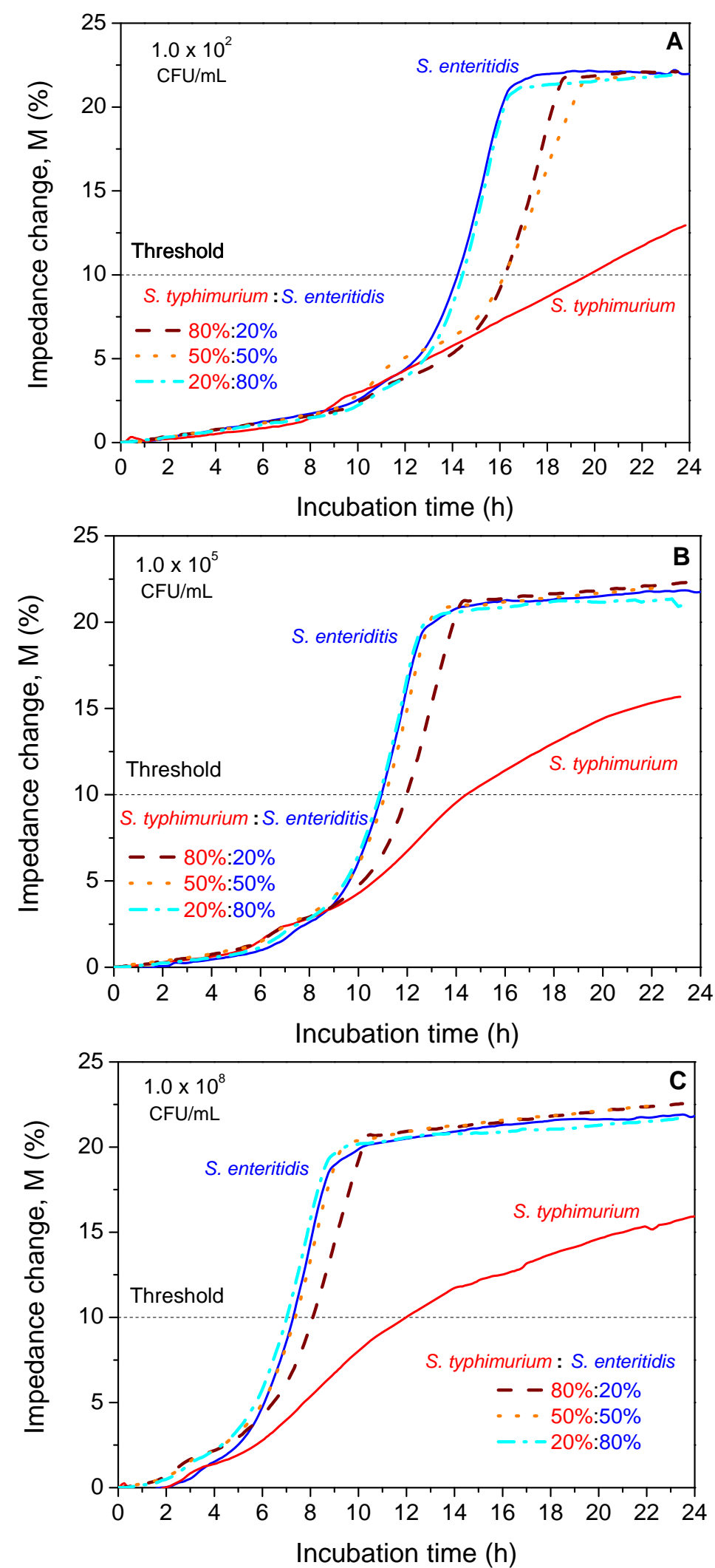

Fig. 4 Impedance growth curves for pure $S$. enteritidis and $S$. typhimurium (solid lines, blue and red, respectively), and mixtures of both strains in a different proportion obtained by diluting from 2 stock solutions from pure Salmonella $\left(1 \times 10^{9}\right.$ CFU/mL) at 3 levels of initial concentration: (a) $1.0 \times 10^{2} \mathrm{CFU} / \mathrm{mL}$; (b) $1.0 \times 10^{5} \mathrm{CFU} / \mathrm{mL}$; (c) $1.0 \times 10^{8} \mathrm{CFU} / \mathrm{mL}$. 

Industry for the Rapid Detection and Quantification of Salmonella Spp.

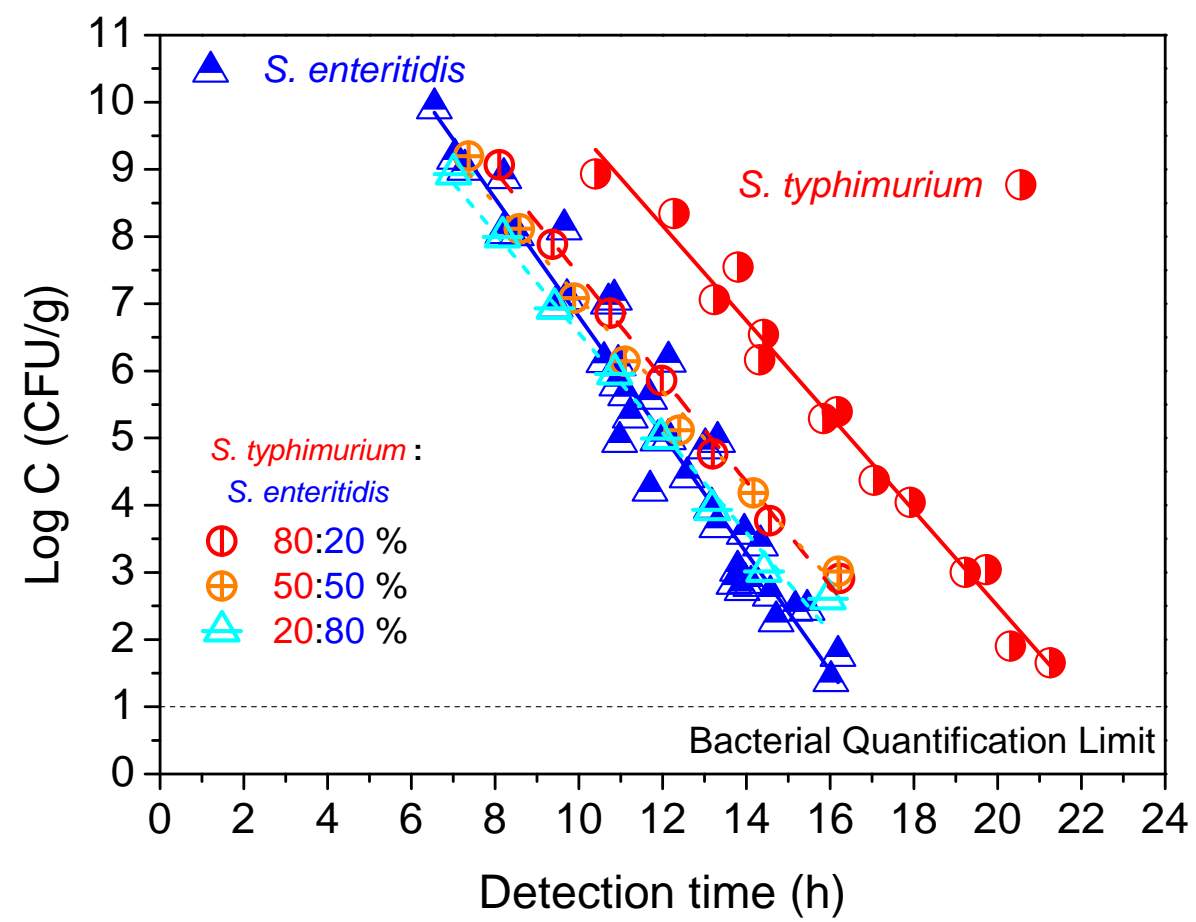

Fig. 5 Logarithmic values of initial concentration of pure $S$. enteritidis and $S$. typhimurium, and mixtures of both strains in a different proportion as a function of detection times obtained from the impedance growth curves ranging from $10^{10} \mathrm{CFU} / \mathrm{g}$ to $10^{1} \mathrm{CFU} / \mathrm{g}$.

Table 1 Values of intercept, slope and correlation coefficient obtained from the linear equation after fitting a regression line by the method of least-squares to the experimental calibrations shown in Fig. $\mathbf{5}$.

\begin{tabular}{llll}
\hline Strain & Intercept (CFU/g) & Slope $(\mathrm{CFU} / \mathrm{g} \cdot \mathrm{h})$ & $\mathrm{R}^{2}$ \\
\hline S. typhimurium (A) & 16.66 & -0.7077 & 0.98 \\
$80 \%$ A:20\% B & 15.15 & -0.7713 & 0.99 \\
$50 \%$ A:50\% B & 14.09 & -0.6995 & 0.99 \\
$20 \%$ A:80\% B & 14.00 & -0.7433 & 0.99 \\
S. enteritidis (B) & 15.60 & -0.8795 & 0.94 \\
\hline
\end{tabular}

by the increase in the values of slope (Table 1), resulting from the linear equation acquired from the calibration curves illustrated in Fig. 5, which provide information of bacterial growth rate too.

Moreover, as viewed in Fig. 5, there are overlapped points from regression lines between the calibration curves of all the mixtures of both Salmonella strains with pure $S$. enteritidis for concentrations higher than $10^{4} \mathrm{CFU} / \mathrm{g}$. It suggests that, despite the delay in $S$. typhimurium growth, when this strain starts its exponential stage of growth, both Salmonella strains seem to grow at the same pace.

Again, values of correlation coefficient higher than 0.9 are obtained as seen in Table 1 , which suggest a strong linear correlation between initial Salmonella concentration and DT for all the cases. Fluctuations are not observed for low concentrations of Salmonella $\left(<2 \log _{10}, \mathrm{CFU} / \mathrm{g}\right)$ either. Therefore, the application of impediometry seems to be a successful tool for rapid quantification of mixtures of different bacterial strains as well.

3.1.4 Validation of Quantification of Salmonella in Raw Pork Meat by Impedance-Based Technology against Standard Plate Count

To validate impedance-based method for quantification of Salmonella in meat, raw pork meat samples were inoculated with $S$. enteritidis at two values of bacterial load corresponding to $10^{4} \mathrm{CFU}$ and 

Industry for the Rapid Detection and Quantification of Salmonella Spp.

$10^{6} \mathrm{CFU}$ and illuminated throughout time with UV-C light. UV-C illumination may be considered as a promising emerging technology which has been reported to be able to reduce the amount of bacteria in food, extending its shelf-life, reducing the probabilities of foodborne illnesses, and therefore, enabling a longer-distance trade [24, 25].

Fig. 6 displays the correlation between the values of concentration of $S$. enteritidis $(\mathrm{CFU} / \mathrm{g})$ in untreated and treated meat samples of initially unknown concentration determined by standard plate count and those predicted by the DT provided by $\mu$-Trac apparatus. On the one hand, the coefficient of variance (CV) was calculated from at least two independent experiments for bacterial levels ranging between 2 and $5 \log _{10}, \mathrm{CFU} / \mathrm{g}$. Values are lower than $15 \%$ for all the levels of bacterial concentrations analysed and for both methods, standard plate count and impediometry. Thus, the degree of variability is considered acceptable since CV values are lower than 20\% [27]. On the other hand, very good agreement in $\log _{10}$ bacterial concentration between count in plates and impedance-based measurements is reached $\left(R^{2}=0.91\right)$. Only differences between results given by plate and impediometry appear for high levels of bacteria since the slope of the regression line becomes higher than 1 . This fact may indicate a higher sensitivity of impediometry $(112 \% \pm 2 \%)$ against standard count plate $(100 \%)$ which is in agreement with Donaghy and Madden [18], Bolton and Gibson [13], Quinn et al. [21], Wawerla et al. [22], Hoorfar [8] and Russell [14]. Moreover, higher errors are attained by standard plate count (horizontal error bars) compared to those of impedance-based method (vertical error bars) which reduces the reproducibility of the quantification assay when using classic microbiology [14, 28]. A more negative effect on plate count accuracy may be due to the presence of natural flora in meat which may hinder the growth of the target microorganism, together with an increase in competence between Salmonella and background flora by nutrients. In contrast, since impedance technique is based on metabolic capacity of the target microorganism, the presence of natural flora may not be such a crucial factor in the accuracy of the

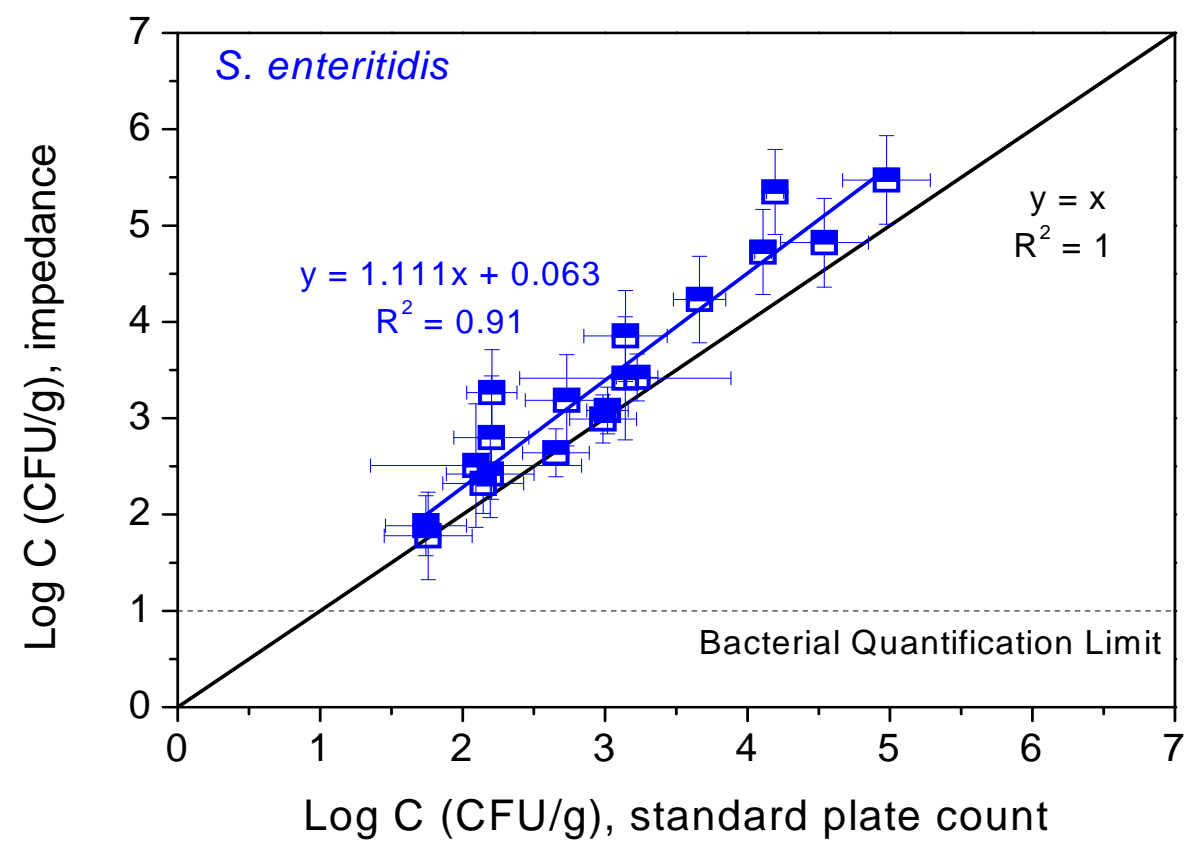

Fig. 6 Concentration of $S$. enteritidis (CFU/g) predicted by the linear equation obtained from the calibration curve given by impedance measurements (Fig. 2) and standard plate count for raw pork meat samples of unknown concentration. Graph function $y=x$ involves a correlation of $100 \%$ in terms of bacterial concentration reported by both methods. Independent experiments have been repeated in triplicate. Experimental points have been collected in duplicate per experiment. Error bars have been calculated from all these repetitions. 

Industry for the Rapid Detection and Quantification of Salmonella Spp.

enumeration method.

Since differences in quantification results between both methods have only been reached at high concentrations, it would not be a problem in food research since those levels of bacteria are not commonly found in food processing industries. It must be pointed out that impedance technique not only exhibits much less error and higher sensitivity for quantifying high levels of bacteria in comparison with plate count, but also, even possible sublethally damaged bacteria by UV-C treatment seem to have been successfully quantified. This observation is opposite to that stated by Wawerla et al. [22], whereas agrees with Russell [14]. This author asserted that despite bacteria may be injured, they are able to produce metabolites as a consequence of their metabolism.

The concentration of Salmonella in logarithmic units present in the treated samples after 5, 15, and 30 min of UV-C illumination have been determined by both methods, standard plate count and impedance changes. The results of the treatment in terms of Salmonella inactivation are depicted in Fig. 7. Similar values in Salmonella concentration acquired by impedance-based technology and standard plate count are achieved. It indicates that quantification of Salmonella in food safety research, e.g. testing the ability of UV-C illumination to inactivate bacteria onto meat samples, can successfully be carried out by impediometry. In addition, apart from reducing time assay compared to that of standard plate count, impedance results can provide much more data in real time, concerning to not only bacterial concentration, but also to the level of activity which is useful in order to know the pathogenic potential of the sample, bacterial kinetics, etc. Those can be useful not only for experimental research but also for predictive microbial modeling.

It must be mentioned that a noticeable reduction in viable concentration of Salmonella, corresponding to 3 orders of magnitude, after 15 min of illumination for $10^{5} \mathrm{CFU} / \mathrm{g}$ and $10^{3} \mathrm{CFU} / \mathrm{g}$ is accomplished. This emerging technology allows reducing the presence of bacteria in food together with the possibility of getting positive samples of Salmonella, fast enough to avoid

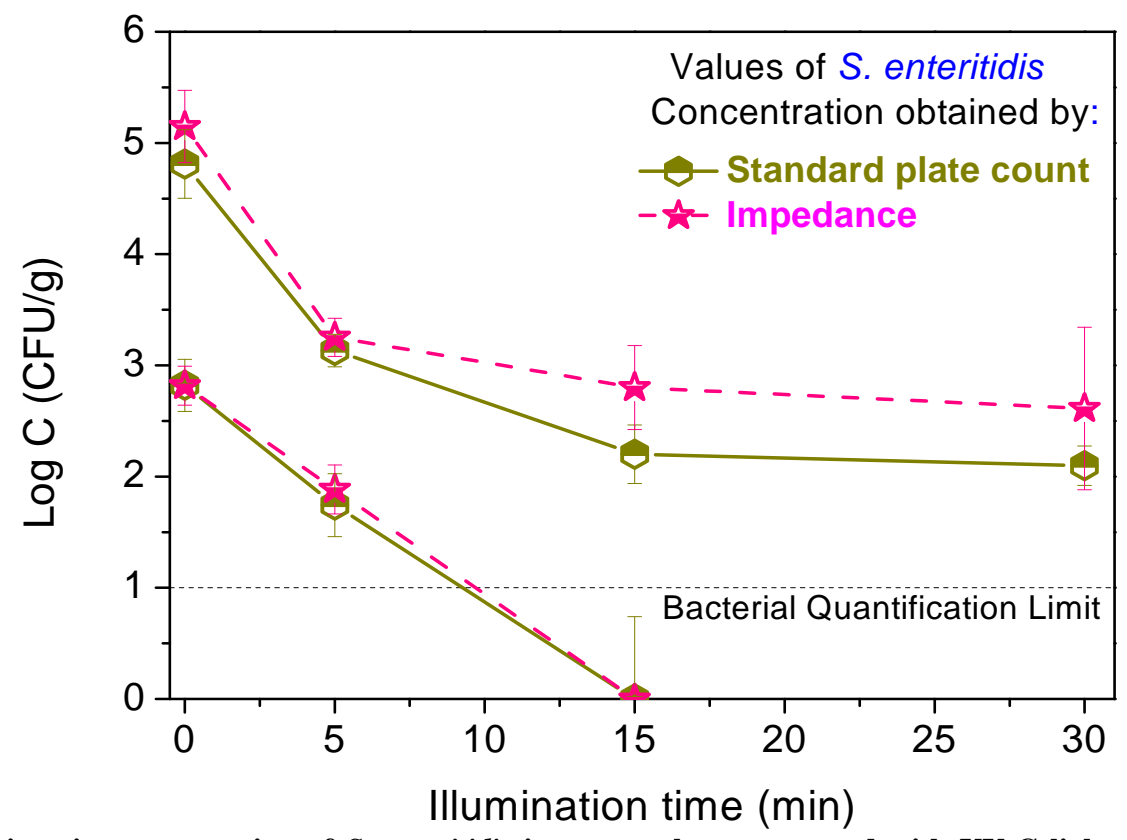

Fig. $7 \log _{10}$ reductions in concentration of $S$. enteritidis in raw pork meat treated with UV-C light. Load of Salmonella previously inoculated onto raw pork meat before UV-C illumination: ca. $10^{4} \mathrm{CFU}$ and $10^{6} \mathrm{CFU}$. Error bars represent the standard deviation. Independent experiments were repeated in triplicate. 

Industry for the Rapid Detection and Quantification of Salmonella Spp.

Table 2 Raw pork meat samples analysed by horizontal method (ISO 6579:2002 Standard) and impedance-based method, for the detection of Salmonella.

\begin{tabular}{|c|c|c|c|c|c|c|}
\hline \multirow[b]{2}{*}{ Sample } & \multicolumn{3}{|c|}{ ISO 6579:2002 Standard } & \multicolumn{3}{|c|}{ Impedance method } \\
\hline & $\begin{array}{l}\text { Plating media } \\
\text { detection }(+/-)^{\mathrm{g}}\end{array}$ & $\begin{array}{l}\text { Biochemical } \\
\text { confirmation } \\
(+/-)\end{array}$ & $\begin{array}{l}\text { Total detection } \\
\text { time }(\mathrm{h})\end{array}$ & $\begin{array}{l}\text { Electrical } \\
\text { impedance, } \\
\text { (h) }\end{array}$ & $\begin{array}{l}\text { Biochemical } \\
\text { DT confirmation } \\
(+/-)\end{array}$ & $\begin{array}{l}\text { Total detection } \\
\text { time }(\mathrm{h})\end{array}$ \\
\hline 1 & - & - & 72 & $-^{c}$ & - & 30 \\
\hline 2 & - & - & 72 & $-^{\mathrm{c}}$ & - & 30 \\
\hline 3 & + & - & 120 & $21.3(+)$ & - & 51.3 \\
\hline 4 & + & - & 120 & $-^{c}$ & - & 30 \\
\hline 5 & - & - & 72 & $-^{\mathrm{c}}$ & - & 30 \\
\hline 6 & - & - & 72 & $-^{\mathrm{c}}$ & - & 30 \\
\hline 7 & - & - & 72 & $-^{\mathrm{c}}$ & - & 30 \\
\hline 8 & + & - & 120 & $-^{\mathrm{c}}$ & - & 30 \\
\hline 9 & + & - & 120 & $-^{\mathrm{c}}$ & - & 30 \\
\hline 10 & + & - & 120 & $21.9(+)$ & - & 51.9 \\
\hline 11 & - & - & 72 & $-^{c}$ & - & 30 \\
\hline $12^{\mathrm{a}}$ & + & + & 120 & $7.4(+)$ & + & 37.4 \\
\hline $13^{\mathrm{b}}$ & + & + & 120 & $6.3(+)$ & + & 36.3 \\
\hline $14^{\mathrm{d}}$ & - & - & 72 & $-^{\mathrm{c}}$ & - & 30 \\
\hline $15^{\mathrm{e}}$ & - & $-^{f}$ & 72 & $-^{\mathrm{c}}$ & $-^{f}$ & 30 \\
\hline False positives (FP) & \multicolumn{3}{|l|}{$5 / 15(33.3 \%)$} & \multicolumn{3}{|l|}{$2 / 15(13.3 \%)$} \\
\hline False negatives (FN) & \multicolumn{3}{|l|}{$0 / 15$} & \multicolumn{3}{|l|}{$0 / 15$} \\
\hline True positives (TP) & \multicolumn{3}{|l|}{$2 / 2$} & \multicolumn{3}{|l|}{$2 / 2$} \\
\hline True negatives (TP) & \multicolumn{3}{|l|}{$2 / 2$} & \multicolumn{3}{|l|}{$2 / 2$} \\
\hline Positives & \multicolumn{3}{|l|}{$2 / 15$} & \multicolumn{3}{|l|}{$2 / 15$} \\
\hline SensitivityTP/(TP+FN) & \multicolumn{3}{|l|}{$100 \%$} & \multicolumn{3}{|l|}{$100 \%$} \\
\hline Specificity TN/(TN+FP) & \multicolumn{3}{|l|}{$100 \%$} & \multicolumn{3}{|l|}{$100 \%$} \\
\hline $\begin{array}{l}\text { Accuracy }(\mathrm{TP}+\mathrm{TN}) / \\
(\mathrm{TN}+\mathrm{TP}+\mathrm{FP}+\mathrm{FN})\end{array}$ & \multicolumn{3}{|l|}{$4 / 9(44.4 \%)$} & \multicolumn{3}{|l|}{$4 / 6(66.7 \%)$} \\
\hline
\end{tabular}

(a) $S$. enteritidis $\left(1 \cdot 10^{4} \mathrm{CFU}\right)$ was previously inoculated in a $25 \mathrm{~g}$ meat sample, positive control.

(b) S. typhimurium $\left(1 \cdot 10^{4} \mathrm{CFU}\right)$ was previously inoculated in a $25 \mathrm{~g}$ meat sample, positive control.

(c) No signal after $24 \mathrm{~h}$.

(d) E. coli $\left(1 \cdot 10^{6} \mathrm{CFU}\right)$ was previously inoculated in a $25 \mathrm{~g}$ meat sample, negative control.

(e) E. faecalis $\left(1 \cdot 10^{6} \mathrm{CFU}\right)$ was previously inoculated in a $25 \mathrm{~g}$ meat sample, negative control.

${ }^{(f)}$ Confirmation test has not carried out since $E$. faecalis is a gram positive bacterium.

(g) Absence (-) and presence (+) of presumptive Salmonella colonies isolated on plating media.

TP: positive test result when Salmonella is present.

TN: negative test result when Salmonella is not present.

FP: positive test result when Salmonella is not present.

FN: negative test result when Salmonella is present.

damage in the external appearance of food, even though high levels of bacteria exist.

3.2 Salmonella Detection Performance in Raw Pork Meat Samples by Impedance Measurements against Traditional Method (ISO 6579:2002 Standard)

The evaluation of impedance technology in comparison with current horizontal method (ISO
6579:2002 Standard) for the detection of Salmonella in samples of raw pork meat appears in Table 2.

AOAC (Association of Official Analytical Chemists) and other validation agencies do not specify acceptance values for sensitivity and specificity but they should be as high as possible [1]. Some authors have reported values of 59.3\%-96.2\% and 92.3\%-97.9\% respectively for other rapid methods such as 

Industry for the Rapid Detection and Quantification of Salmonella Spp.

enzyme-immunological and nucleic acid-based assays [7]. Statistical parameters such as sensitivity, also called the true positive rate (proportion of actual positive samples correctly identified by the method as positives); specificity, also called the true negative rate (proportion of actual negative samples correctly detected by the method as negative) [31]; and accuracy (closeness of a measurement to the true value) have been determined.

Both methods provide $100 \%$ sensitivity (when 2 strains of Salmonella were previously inoculated in two samples) and $100 \%$ specificity (when 2 strains different from Salmonella, E. coli and E. faecalis, were inoculated). This indicates that the alternative method is adequate detecting true positive and true negative samples. It must be worth noticing that any of the methods report false negative results. However, the impedance-based method exhibits a higher accuracy $(66.7 \%)$ compared to the standard method (44.4\%) as well as a lower rate of false positive out of 15 , corresponding to $13.3 \%(2 / 15)$ in comparison with $33.3 \%(5 / 15)$ obtained for the conventional method. In general, a higher number of false negatives and positives are identified for the conventional method according to Quinn et al. [21], and Wawerla et al. [22]. It may be due to natural flora [1] present in meat sample which may either hinder the growth of the target microorganism or lead to misleading results as happens for Proteus spp. Actually, Proteus mirabilis and P. vulgaris together with Citrobacter freundi were identified in the biochemical confirmation test for presumptive Salmonella-positive samples. Therefore, accuracy and efficacy may be lower than that of rapid detection methods [7].

In addition, the impedance method noticeably reduces the total time of the Salmonella detection assay since a negative result will be obtained between $30 \mathrm{~h}(6 \mathrm{~h}$ of pre-enrichment following the impedance measure for $24 \mathrm{~h}$ ) up to $54 \mathrm{~h}$ in case a biochemical confirmation is required. Positive samples can be detected between 8 and less than $30 \mathrm{~h}$, before carrying out the biochemical test.

Impedance method studied in the present work seems to be very suitable as a screening test for the pork meat processing industry. Using the impedance method, the total time for detection of Salmonella in negative meat samples can be decreased from 42 up to $66 \mathrm{~h}$, in comparison with the standard method. In view of the time of analysis, this method may be even superior to the enzyme-immunological and nucleic acid-based tests whose detection times range between 38 to $72 \mathrm{~h}$ due to the necessity of longer pre-enrichment periods.

\section{Conclusions}

High values of correlative coefficients obtained from calibration curves of different strains of Salmonella in raw pork meat together with good correlation between impediometry and standard plate counting to compare the reduction of microbial load throughout UV-C illumination of pork meat samples show that impedance technology represents an approach to quantitative microbiology. Although a calibration curve is required per bacteria and matrix prior to performing routine food assays, it would not be a shortcoming for food safety research as a high percentage of similar samples are used. This fact along with the possibility of monitoring impedance bacterial growth curves in real time gives rise to different applications to be developed in food safety research. As novelty confirmed by experimentation shown in this work, not only low bacterial detection and quantification limits have been reached, but also damaged but still viable bacteria can successfully be detected. Both facts are significantly important in experimentation concerning to emerging technologies as they exert mechanisms of stress for inactivating bacteria.

Efficiency of impedance technology used as tool for detection of Salmonella has also been proven as the unnecessarily rejection of batches is avoided due to its ability to distinguish between live and dead cells. In 

Industry for the Rapid Detection and Quantification of Salmonella Spp.

addition, impediometry yields a negative result within $30 \mathrm{~h}$ compared to $72 \mathrm{~h}$ required for conventional method, enabling to release negative batches in a faster and safer way.

\section{Acknowledgements}

The authors gratefully acknowledge the financial support of the Ministerio de Ciencia e Innovación (MICINN) of Spain through the project WATER4FOOD (CTQ2014-54563-C3-1-R) and Comunidad de Madrid through the program REMTAVARES (S2013/MAE-2716) and Embutidos La Nuncia S.L., local meat processing industry.

\section{References}

[1] Lee, K. M., Runyon, M., Herrman, T. J., Phillips, R., and Hsieh, J. 2015. "Review of Salmonella Detection and Identification Methods: Aspects of Rapid Emergency Response and Food Safety." Food Control 47: 264-76.

[2] Yang, L., and Bashir, R. 2008. "Electrical/Electrochemical Impedance for Rapid Detection of Foodborne Pathogenic Bacteria." Biotechnol Adv. 26: 135-50.

[3] Hugas, M., and Tsigarida, E. 2008. "Pros and Cons of Carcass Decontamination: The Role of the European Food Safety Authority." Meat Sci. 78: 43-52.

[4] European Union. 2005. "Commission Regulation (EC) No 2073/2005 of 15 November 2005 on Microbiological Criteria for Foodstuffs." Official Journal of the European Union.

[5] European Union. 2007. "Commission Regulation (EC) No 1441/2007 of 5 December 2007 Amending Regulation (EC) No 2073/2005 on Microbiological Criteria for Foodstuffs." Official Journal of the European Union.

[6] ISOUNE-EN ISO 6579:2002 Standard. 2002. "Microbiology of Food and Animal Feeding Stuffs. Horizontal Method for the Detection of Salmonella spp." AENOR (Spanish Association for Standardization and Certification).

[7] Van der Zee, H., and Huis in'tVeld, J. H. J. 2000. Salmonellain Domestic Animals, edited by Wray, C., and Wray, A., 1st ed. New York: CABI Publishing, 373-92.

[8] Hoorfar, J. 2011. "Rapid Detection, Characterization, and Enumeration of Foodborne Pathogens." Acta. Pathol. Mic. Sc.119 (Suppl. 133): 1-24.

[9] Russell, S. M. 2004-2005. "Salmonella and Campylobacter: A Methods Update." Food Safety Magazine 10 (6) (December 2004/January 2005) [Online], http://www.foodsafetymagazine.com/magazine-archive1/ december-2004january-2005/salmonella-and-campylobac ter-a-methods-update/. Accessed May 5, 2016.

[10] Bedale, W. 2015. "Meeting Review: Rapid Methods in Food Safety Microbiology." Food Protection Trends 35 (2): 130-5.

[11] Ruan, C., Yang, L., and Li, Y. 2002. "Rapid Detection of Viable Salmonella Typhimurium in a Selective Medium by Monitoring Oxygen Consumption with Electrochemical Cyclic Voltammetry." J. Electroanal. Chem. 519: 33-8.

[12] Blivet, D., Salvat, G., Humbert, F., and Colin, P. 1998. "Development of a New Culture Medium for the Rapid Detection of Salmonella by Indirect Conductance Measurements." J. Appl. Microbiol. 84: 399-403.

[13] Bolton, F. J., and Gibson, B. M. 1994. Rapid Analysis Techniques in Food Microbiology, 1st ed. Edited by Patel, P. Glasgow: Chapman \& Hall, 131-69.

[14] Russell, S. M. 2003. "Advances in Automated Rapid Methods for Enumerating E. coli." Food Safety Magazine (February/March 2003) [Online], http://www.foodsafetymagazine.com/magazine-archive1/ februarymarch-2003/advances-in-automated-rapid-metho ds-for-enumerating-colii/. Accessed May 05, 2016.

[15] Richards, J. C. S., Jason, A. C., Hobbs, G., Gibson, D. M., and Christie, R. H. 1978. "Electronic Measurement of Bacteria Growth.” J. Phys. E. Sci. Instrum 11: 560-8.

[16] Eden, R., and Eden, G. 1984. Impedance Microbiology. Hertfordshire: Research Studies Press Ltd.

[17] Owens, J. D., Thomas, D. S., Thompson, P. S., and Timmerman. J. W. 1989. "Indirect Conductimetry: A Novel Approach to the Conductimetric Enumeration of Microbial Populations." Lett. Appl. Microbiol 9: 245-9.

[18] Donaghy, J. A., and Madden, R. H. 1993. "Detection of Salmonella in Animal Protein by Rappaport-Vassiliadis Broth Using Indirect Impediometry." Int. J. Food Microbiol 17 (4): 281-8.

[19] Jockel, J. 1996. "Use of Impedance Method in Official Food Inspection.” Fleischwirtschaft 76 (9): 945-50. (in German)

[20] Pless, P., Futschik, K., and Schopf, E.1994. "Rapid Detection of Salmonellae by Means of a New Impedance-Splitting Method.” J. Food Protect 57 (5): 369-76.

[21] Quinn, C., Ward, J., Griffin, M., Yearsley, D., and Egan, J. 1995. "A Comparison of Conventional Culture and Three Rapid Methods for the Detection of Salmonella in Poultry Feeds and Environmental Samples." Lett. Appl. Microbiol 20 (2): 89-91.

[22] Wawerla, M., Stolle, A., Schalch, B., and Eisgruber. H. 1999. "Impedance Microbiology: Applications in Food Hygiene.” J. Food Protect 62 (12): 1488-96. 

Industry for the Rapid Detection and Quantification of Salmonella Spp.

[23] Gibson, D. M., Coombs, P. C., and Pimbley, D. W.1992. "Automated Conductance Method for the Detection of Salmonella in Foods: Collaborative Study." JAOAC Int. 75: 293-7.

[24] Aymerich, T., Picouet, P. A., and Monfort, J. M. 2008. "Decontamination Technologies for Meat Products." Meat Sci. 78: 114-29.

[25] Bintsis, T., Litopoulou-Tzanetaki, E., and Robinson, R. K. 2000. "Existing and Potential Applications of Ultraviolet Light in the Food Industry-A Critical Review." J. Sci. Food Agr. 80: 637-45.

[26] Fehlhaber, K., and Krüger, G. 1998. "The Study of Salmonella Enteritidis Growth Kinetics Using Rapid Automated Bacterial Impedance Technique.” J. Appl. Microbiol 84: 945-9.

[27] Johnson, N., Chang, Z., Bravo Almeida, C., Michel, M., Iversen, C., and Callanan, M. 2014. "Evaluation of Indirect Impedance for Measuring Microbial Growth in Complex Food Matrices.” Food Microbiol 42: 8-13.
[28] Koutsoumanis, K., Tassou, C. C., Taoukis, P. S., and Nychas, G. J. E. 1998. "Modelling the Effectiveness of a Natural Antimicrobial on Salmonella Enteritidis as a Function of Concentration, Temperature and $\mathrm{pH}$, Using Conductance Measurements." J. Appl. Microbiol 84: 981-7.

[29] UNE-EN ISO 16140:2003 Standard. 2003. "Microbiology of Food and Animal Feeding Stuffs-Protocol for the Validation of Alternative Methods." AENOR (Spanish Association for Standardization and Certification).

[30] Yang, L., Banada, P. P., Liu, Y. S., Bhunia, A. K., and Rashid, B. 2005. "Conductivity and pH Dual Detection of Growth Profile of Healthy and Stressed Listeria Monocytogenes." Biotechnol Bioeng 92 (6): 685-94.

[31] Jawad, G. M., Marrow, T., and Odumeru, J. A. 1998. "Assessment of Impedance Microbiological Method for the Detection of Escherichia Coli in Foods." J. Rapid Meth Aut. Mic. 6: 297-305. 